\title{
Guida alla lettura dei risultati della metanalisi dello studio: "Medical management to prevent recurrent nephrolithiasis in adults: a systematic review for an American College of Physicians Clinical Guideline"
}

\author{
Commento all'articolo: "Medical management to prevent recurrent nephrolithiasis in adults: \\ a systematic review for an American College of Physicians Clinical Guideline”
}

\section{Anteo Di Napoli, Michele Nichelatti}

Registro Italiano Dialisi e Trapianto

Lo studio in questione si proponeva di valutare le problematiche relative ai benefici e ai rischi di trattamenti per la prevenzione clinica della nefrolitiasi recidivante negli adulti, attraverso una revisione sistematica della letteratura e la conduzione di una metanalisi per la valutazione degli effetti.

L'incidenza di calcolosi renale è pari a circa il 13\% tra i maschi e al 7\% tra le donne. Il riscontro occasionale di calcoli è osservato in circa il 5\% degli individui che si sottopongono a esami ecografici o a TC. Tra 1'11 e il 32\% dei soggetti con calcoli ma asintomatici, seguiti con esami radiografici ripetuti, sviluppano sintomi o vanno incontro a procedure chirurgiche entro 3-4 anni. Inoltre, il tasso di recidiva a 5 anni da un episodio di calcolosi sintomatica non trattato è tra il $35 \%$ e il $50 \%$.

Le strategie di trattamento si propongono di prevenire il verificarsi di calcoli renali attraverso un miglioramento del bilancio urinario tra sostanze che favoriscono e inibiscono la formazione di cristalli (principalmente ossalati e fosfati di calcio). Non essendo chiari i benefici e i rischi dei trattamenti disponibili e come misurarne gli esiti anche attraverso indicatori biochimici, è stata condotta una revisione sistematica finalizzata a produrre linee guida cliniche per la prevenzione della nefrolitiasi nell'adulto destinate all'American College of Physicians.

La revisione sistematica e la metanalisi sono state condotte secondo i principi operativi della Cochrane Collaboration, un grande network internazionale non-profit costituito da numerosi gruppi collaborativi che producono e diffondono metanalisi di trattamenti relativi a specifici problemi sanitari. Le metanalisi della Cochrane Collaboration sono raccolte nella Cochrane Library e disponibili sul sito del network (sito www.cochrane.org).

Le revisioni debbono essere elaborate con criteri metodologici rigorosi e precedute dall'approvazione, ad opera di esperti, del protocollo di ricerca (peer review), definendo il razionale della ricerca con i suoi endpoint principali e secondari. Quindi vanno definiti i criteri di inclusione ed esclusione degli studi, i metodi statistici impiegati e le loro assunzioni nell'eventuale successiva metanalisi; i criteri per valutare la consistenza dei risultati; la modalità di presentazione dei risultati.

Questi criteri sembrerebbero essere stati seguiti dagli Autori del presente studio, i quali hanno pubblicato in maniera esaustiva la strategia di conduzione della revisione sistematica condotta a partire da un protocollo elaborato con il contributo di tutti i portatori d'interesse. In particolare, sono state illustrate: le domande chiave cui rispondere per l'elaborazione della linea guida (Appendix Figure 1); le fonti utilizzate e la strategia di ricerca che ha portato a selezionare gli RCT (Appendix Table I); i criteri di eleggibilità degli studi (Appendix Table II); la descrizione della qualità dei singoli studi (Appendix Table III); la descrizione dei criteri di classificazione della forza delle evidenze per la prevenzione della recidiva di calcolosi (Appendix Table IV).

In particolare, gli Autori hanno stabilito di selezionare studi esclusivamente in lingua inglese che riguardavano dieta o terapia farmacologica per la prevenzione della nefrolitiasi nell'adulto e riportavano esiti clinici (identificati attraverso la sintomatologia o segni radiologici) di recidiva dei calcoli renali o modifiche della loro dimensione.

Una flow-chart della strategia di selezione e ricerca degli RCT da inserire nella revisione è stata presentata nella Appendix Figure 2. La strategia è stata estremamente selettiva (ad esempio esclusione degli studi non pubblicati in lingua inglese), il che può aver introdotto dei bias, oltre a quelli di per sé annessi a ogni ricerca bibliografica, legati al bias di pubblicazione (tendenza a pubblicare solo RCT con risultati significativi).

La forza delle evidenze relativa a interventi di tipo dietetico o farmacologico e un riepilogo delle evidenze è stato riassunto nelle 
Appendix Tables V-VIII.

La valutazione qualitativa, condotta attraverso criteri espliciti, della metodologia utilizzata dai singoli studi è alla base della loro selezione per una metanalisi, che prevede l'estrazione dei dati dai singoli studi e, quando possibile, la loro combinazione statistica secondo opportune metodiche.

La valutazione qualitativa deve considerare non pochi aspetti problematici della strategia di revisione sistematica. Le caratteristiche dei pazienti arruolati potrebbero differire tra studio e studio, indipendentemente dall'eventuale diagnosi comune. I trattamenti valutati pur essendo i medesimi, in realtà potrebbero differire per aspetti difficili da definire (tipo specifico, durata e posologia, via di somministrazione, associazione con altri farmaci, effetti collaterali). Il setting nel quale le sperimentazioni vengono condotte può differire da studio a studio in maniera tale da rendere poco generalizzabili e quindi non confrontabili i risultati dei singoli studi selezionati.

Una metanalisi completa l'approccio fondamentalmente narrativo di una revisione sistematica della letteratura, con una metodologia di tipo statistico. Tuttavia, la combinazione di studi qualitativamente dissimili può inficiare la qualità dei risultati della metanalisi.

Il principale vantaggio di una metanalisi rispetto a uno studio singolo risiede nella possibilità di combinare quantitativamente i dati di più studi, consentendo di aumentare la potenza statistica, il che si riflette innanzitutto sul miglioramento della precisione nella stima di un effetto. Si consideri che gli studi pubblicati non di rado sono sottodimensionati, includendo un numero di soggetti insufficiente a dimostrare in maniera statisticamente significativa l'efficacia di un trattamento.

Tuttavia, paradossalmente, proprio la possibilità di aumentare la potenza dei singoli studi, analizzandone i dati in maniera combinata può determinare il problema opposto; la disponibilità di numerosità campionarie molto grandi può far sì che la significatività statistica sull'efficacia di un trattamento possa essere più facilmente raggiunta, senza che questo ovviamente equivalga a una dimostrata significatività clinica del risultato osservato.

In termini quantitativi le informazioni necessarie per condurre una metanalisi sono molto semplici, riassumibili in una classica Tabella 2x2 ricavabile da ciascuno degli studi considerati, dove si possa verificare chi ha sperimentato o meno l'esito in studio (ad esempio, morti $v s$ vivi) rispetto al gruppo considerato (ad esempio, trattati $v s$ controlli). Ogni studio viene considerato separatamente, attraverso un'analisi stratificata per singolo studio che valuti l'effetto del trattamento prima per ciascuno di essi; solo dopo i singoli effetti studio-specifici vengono combinati per stimare l'effetto complessivo, assegnando dei pesi opportuni ai risultati dei singoli studi.

I metodi statistici che si utilizzano sono riassumibili in due tipologie di modello: a effetti fissi e a effetti random.

Il modello di metanalisi a effetti fissi assume che non vi sia eterogeneità tra gli studi e che le differenze osservate tra loro siano ascrivibili al caso. Tale modello calcola una statistica riassuntiva (metodi di Mantel-Haenszel) della misura di effetto e della sua varianza come media pesata dei singoli studi, dove i pesi sono funzione esclusivamente dalla variabilità delle singole stime; evidentemente una diversa definizione dei pesi produce stime diverse. I dati si classificano confrontando "osservati" (pazienti che hanno manifestato l'evento in studio) e "attesi" (pazienti che dovrebbero manifestare l'evento in studio se il trattamento fosse del tutto inefficace). Operativamente si procede come per il calcolo del Chi quadrato. È importante sottolineare che questa metodologia preserva l'individualità dei singoli studi, in quanto non sono i pazienti dei singoli studi ad essere sommati come se si trattasse di un megastudio, ma si combinano le stime degli effetti di ciascuno studio.

Il modello di metanalisi a effetti random assume esplicitamente l'esistenza di eterogeneità tra studi diversi, considerando oltre alle differenze casuali nelle stime studio-specifiche anche una fonte aggiuntiva di variabilità data dalla eterogeneità dell'effetto del trattamento tra gli studi e, quindi, ne tiene conto incorporando la stima dell'eterogeneità nei pesi assegnati. In definitiva, nel modello a effetti random si assume che il valore atteso della stima di ogni singolo studio sia uguale alla stima globale dell'effetto del trattamento, mentre la varianza della stima di effetto in ogni singolo studio misura sia la variazione dell'effetto del trattamento intra-studio, sia la variazione dell'effetto del trattamento inter-studi. Più è elevato il valore dell'eterogeneità, più le stime studio-specifiche sono diverse e la stima congiunta a effetti casuali diverge dalla stima a effetti fissi; più basso il valore dell'eterogeneità, più le stime di associazione tra trattamento ed esito dei diversi studi sono omogenee e la stima a effetti casuali si avvicina a quella a effetti fissi.

La stima della varianza tra studi viene effettuata attraverso il metodo di DerSimonian e Laird, che si basa sulla statistica test Q di omogeneità, la quale testa l'ipotesi nulla di omogeneità delle stime studio-specifiche, e i relativi p-value sono stati calcolati sulla base della distribuzione Chi2 di Q, con numero di gradi di libertà pari al numero degli studi meno 1 . Solitamente, per tutte le stime di una metanalisi si riporta il p-value di eterogeneità ottenuto dalla distribuzione della statistica Q; un p-value di eterogeneità superiore a 0.05 supporta l'ipotesi nulla di uguaglianza delle singole stime (omogeneità tra studi); viceversa un p-value di eterogeneità inferiore a 0.05 supporta l'ipotesi alternativa di eterogeneità delle stime studio-specifiche.

La scelta tra modello a effetti fissi e modello a effetti random dipende dalla eterogeneità degli studi. Se si presume che l'eterogeneità sia trascurabile e quindi in sostanza ciascuno studio misura lo stesso effetto comune è preferibile il modello a effetti fissi che non prevedendo aggiustamento per eterogeneità, ma solo una pesatura sulla numerosità degli studi, produce intervalli di confidenza più stretti.

Quando, invece, si ritiene che la dispersione dei risultati sia tale da evidenziare una eterogeneità tra i singoli studi la stima di un unico effetto del trattamento può essere distorta. In tal caso è preferibile ricorrere al modello a effetti random che prevede di stimare l'ipotetico effetto medio attraverso un range di risultati possibili. Il tener conto dell'eterogeneità fa sì che la stima 
combinata dell'effetto sia più vicina alla media delle singole stime e presenti intervalli di confidenza più ampi. Se l'assunzione di eterogeneità non è necessaria è preferibile evitare l'utilizzo del modello a effetti random che prevede calcoli più complicati e si avvale di un test statistico meno efficiente.

Nella metanalisi del presente studio gli Autori dichiarano di aver utilizzato modelli a effetti random per generare stime combinate dei rischi relativi. In realtà, vista la bassa eterogeneità misurata tra gli effetti dei singoli studi avrebbero potuto avvalersi di un modello a effetti fissi.

Gli Autori hanno valutato il rischio di recidiva di calcolo renale (sintomatico o diagnosticato radiologicamente), confrontando gli effetti del trattamento rispetto al placebo o a terapia di controllo, ai quali i pazienti erano stati assegnati in maniera random. Gli Autori hanno riassunto i risultati nei classici "Forest-plot", presentati in Figura, con i quali si illustrano i risultati di una metanalisi (il logo della Cochrane Collaboration è proprio un "Forest-plot").

In esse, sono riportate sulla linea orizzontale i valori puntuali dei rischi relativi (in scala logaritmica, al fine di avere intervalli di confidenza simmetrici). Al centro della linea orizzontale è segnato il valore che indica l'equivalenza fra i due trattamenti (in questo caso 1, poiché si tratta di una misura di rapporto tra rischi). Le stime puntuali sono rappresentate sotto forma di quadrati, la cui superficie varia a seconda del peso dello studio nella metanalisi, e sono accompagnate dai rispettivi intervalli di confidenza (ampiezza della linea corrisponde a quella dell'intervallo). La precisione della misura dell'effetto terapeutico è inversamente proporzionale all'ampiezza dell'intervallo di confidenza; quando l'intervallo di confidenza attraversa la linea di equivalenza verticale, l'effetto terapeutico non è statisticamente significativo. I risultati dei singoli trials e della loro combinazione (rappresentata come un rombo), sono ordinati perpendicolarmente alla linea verticale di equivalenza. Nella metanalisi in questione tutte le stime sono collocate a sinistra della linea verticale di equivalenza, indicano un rischio relativo più basso nei trattati rispetto al placebo o ai controlli. Se tali risultati siano stati influenzati da criteri di selezione troppo rigidi degli studi è questione aperta.

Nella Figura sono anche esplicitati per ogni studio: il numero di eventi osservati sul totale dei soggetti randomizzati a uno dei due bracci del trial; il peso (espresso come percentuale) dello studio nella metanalisi; i valori di rischio relativo con intervallo di confidenza, per i singoli trials e complessivo per la metanalisi. Inoltre sono riassunte le misura dell'eterogeneità tra studi e il test che misura la significatività dell'effetto del trattamento, calcolato come misura complessiva di rischio relativo su tutti gli studi della metanalisi.

Dichiarazione di conflitto di interesse: Gli Autori dichiarano di non avere conflitto di interessi.

Contributi economici agli Autori: Gli Autori dichiarano di non avere ricevuto sponsorizzazioni economiche per la preparazione dell'articolo.

Indirizzo degli Autori:

Dr. Anteo Di Napoli

anteo.dinapoli@libero.it

Dr. Michele Nichelatti

michele.nichelatti@ospedaleniguarda.it

\section{Letture consigliate}

- $\quad$ Fink HA, Wilt TJ, Eidman KE, et al. Medical management to prevent recurrent nephrolithiasis in adults: a systematic review for an American College of Physicians Clinical Guideline. Ann Intern Med 2013; 158(7): 535-43.

- $\quad$ Banzi R, Moja L, Pistotti V, Facchini A, Liberati A. Conceptual frameworks and empirical approaches used to assess the impact of health research: an overview of reviews. Health Res Policy Syst 2011; 9: 26.

- $\quad$ Bero LA, Rennie D. The Cochrane Collaboration. Preparing, maintaining and disseminating systematic reviews of the effects of health care. JAMA 1995; 274: 1935-8.

- $\quad$ Brunetti M, Shemilt I, Pregno S, Vale L, Oxman AD, Lord J, Sisk J, Ruiz F, Hill S, Guyatt GH, Jaeschke R, Helfand M, Harbour R, Davoli M, Amato L, Liberati A, Schünemann HJ. GRADE guidelines: 10. Considering resource use and rating the quality of economic evidence. J Clin Epidemiol 2013; 66(2):140-50.

- DerSimonian R, Laird N. Meta-analysis in clinical trials. Control Clin Trials 1986;7: 177-88.

- $\quad$ Egger M, Smith GD. Meta-analysis. Potentials and promise. BMJ 1997; 315: 1371-4.

- Guyatt GH, Oxman AD, Kunz R, Vist GE, Falck-Ytter Y, Schünemann HJ; GRADE Working Group. What is "quality of evidence" and why is it important to clinicians? BMJ 2008; 336(7651): 995-8.

- Guyatt GH, Oxman AD, Vist GE, Kunz R, Falck-Ytter Y, Alonso-Coello P, Schünemann HJ; GRADE Working Group. GRADE: an emerging consensus on rating quality of evidence and strength of recommendations. BMJ 2008; 336(7650): 924-6.

- Hunt D, McKibbon KA. Locating and appraising systematic reviews. Ann Intern Med 1997; 126: 532-8. 
- $\quad$ Ioannidis JP, Cappelleri JC, Lau J. Issues in comparison between meta-analyses and large trials. JAMA 1998; 279: $1089-93$.

- Ioannidis JP, Lau J. Can quality of clinical trials and meta-analyses be quantified? Lancet 1998; 352(9128): 590-1.

- Lau J, et al. Cumulative meta-analysis of clinical trials builds evidence for exemplary medical care. J Clin Epidemiol 1995; 48 : 45-57.

- LeLorier J, et al. Discrepancies between meta-analyses and subsequent large randomized controlled trials. N Engl J Med 1997; 337: 536-42.

- Liberati A, Altman DG, Tetzlaff J, et al. The PRISMA statement for reporting systematic reviews and meta-analyses of studies that evaluate healthcare interventions: explanation and elaboration. BMJ 2009; 339: b2700.

- Liberati A, Altman DG, Tetzlaff J, et al. The PRISMA statement for reporting systematic reviews and meta-analyses of studies that evaluate health care interventions: explanation and elaboration. J Clin Epidemiol 2009; 62(10): e1-34.

- Liberati A, D'Amico R. Commentary: The debate on non-inferiority trials: 'when meta-analysis alone is not helpful'. Int J Epidemiol 2010; 39(6): 1582-3.

- Liberati A. The Cochrane collaboration as key player in the struggle for better and more relevant patients- and system-oriented research. Epidemiol Psychiatr Sci 2011; 20: 225-30.

- Moher D, Liberati A, Tetzlaff J, Altman DG; PRISMA Group. Preferred reporting items for systematic reviews and meta-analyses: the PRISMA statement. J Clin Epidemiol 2009; 62(10):1006-12.

- Moher D, Liberati A, Tetzlaff J, Altman DG; PRISMA Group. Preferred reporting items for systematic reviews and meta-analyses: the PRISMA statement. BMJ 2009; 339:b2535.

- Moher D, Liberati A, Tetzlaff J, Altman DG; PRISMA Group. Preferred reporting items for systematic reviews and meta-analyses: the PRISMA statement. Int J Surg 2010; 8(5): 336-41.

- Moja L, Moschetti I, Liberati A, Gensini GF, Gusinu R. Understanding systematic reviews: the meta-analysis graph (also called 'forest plot'). Intern Emerg Med 2007; 2(2): 140-2.

- $\quad$ Rothwell PM. Can overall results of clinical trials be applied to all patients? Lancet 1995; 345: 1616-9.

- Stafoggia M, Colais P, Serinelli M; Gruppo collaborativo EpiAir. [Methods of statistical analysis to evaluate the short term effects of air pollution in the EpiAir Project]. Epidemiol Prev 2009; 33(6 Suppl 1): 53-63.

- Thompson SG. Why sources of heterogeneity in meta-analysis should be investigated. BMJ 1994; 309: 1351-5. 